\title{
A market regulation bilevel problem: a case study of the Mexican petrochemical industry
}

\author{
Héctor Maravillo ${ }^{\mathrm{a}}$, José-Fernando Camacho-Vallejo ${ }^{\mathrm{a}, *}$, Justo Puerto ${ }^{\mathrm{b}}$, Martine Labbéc $^{\mathrm{c}}$ \\ ${ }^{a}$ Facultad de Ciencias Físico-Matemáticas, Universidad Autónoma de Nuevo León, Monterrey, Mexico \\ ${ }^{b}$ Department of Statistics and Operational Research \& Instituto de Matemáticas Universidad de Sevilla, \\ Universidad de Sevilla, Seville, Spain \\ ${ }^{c}$ Départament d'Informatique, Université Libre de Bruxelles, Brussels, Belgium and INRIA, Lille, France
}

\begin{abstract}
In this paper, a bilevel programming model is proposed to study a problem of market regulation through government intervention. One of the main characteristics of the problem herein analyzed is that the government monopolizes the raw material in one industry, and competes in another industry with private firms for the production of commodities. Under this scheme, the government controls a state-owned firm to balance the market; that is, to minimize the difference between the produced and demanded commodities. On the other hand, a regulatory organism that coordinates private firms aims to maximize the total profit by deciding the amount of raw material bought from the state-owned firm. Two equivalent single-level reformulations are proposed to solve the problem. The first reformulation is based on the strong duality condition of the lower level and results in a continuous nonlinear model. The second reformulation resorts to the complementarity slackness optimality constraints yielding a mixed-integer linear model. Additionally, three heuristic algorithms are designed to obtain good-quality solutions with low computational effort. In this problem, the feasible region of the dual problem associated to the follower is independent from the leader's decision. Therefore, the proposed heuristics exploit this particular characteristic of the bilevel model. Moreover, the third heuristic hybridizes the other two algorithms to enhance its performance. Extensive computational experimentation is carried out to measure the efficiency of the proposed solution methodologies. A case study based on the Mexican petrochemical industry is presented. Additional instances generated from the case study are considered to validate the robustness of the proposed heuristic algorithms. Numerical results indicate that the hybrid algorithm outperforms the other two heuristics. However, all of them demonstrate to be good alternatives for solving the problem. Additionally, optimal solutions of all the instances are obtained by using good quality solutions (given by the hybrid algorithm) as initial solutions when solving the second reformulation via a general purpose solver.
\end{abstract}

Keywords: Market regulation, Bilevel programming, Petrochemical industry 


\section{Introduction}

Market regulation through government intervention has appeared in different ways, such as, fiscal policies (taxes and subsidies), anti-monopoly legislation, price control, quantity control, and nationalization of firms [1]. Two of the main motivations for regulating the economy is to seek social equity and to handle market failures [2]. A specific manner of government intervention is through the participation of state-owned firms, which are under the government control and compete with the private firms. The goal to regulate the market by participation has been very noticeable in developing countries, mainly in raw materials industries [3]. For instance, a list of countries in which market regulation by participation exists in oil industry is presented in [4], [5], [6]. At this point we would like to emphasize that this situation occurred in Mexico in the petrochemical industry from 1958 until 2014.

The first paper that considers the idea of regulate the market by including a state-owned firm is [7]. In that paper, a short-term analysis that concerns with the entrance of a stateowned firm into a three-firms oligopoly market is done. They conclude that the existence of a state-owned firm may improve the performance of the market, which it is also shown in [8] in the aerospace market. Following up with the existence of state-owned firms, [9] considers a situation in which the difference between the production and the production level is made up by the government. The novelty in that paper is to realize that state-owned firm acts as the dominant decision-maker, that is, it has complete information of the market and announces its decision. Hence, each private firm reacts to this decision by establishing its output level so that its marginal cost equals the price. Furthermore, [10] explicitly considers the state-owned firm as a leader in a Stackelberg game. Later, in [11], a mixed oligopoly model that helps to compare the differences among the nationalization of a state-owned firm and the entrance of a new state-owned firm is studied. This last paper also analyzes the cost effectiveness of the state-owned firms in the long-term and moreover, the authors consider for the first time a budgetary constraint associated with the state-owned firm to guarantee a minimum profit.

Literature reviews regarding mixed oligopolies can be found in [12] and [13]. In those reviews the common characteristics of these models are identified. Particularly, the context of the problem, the cases when the government provides complete information, the goal of the state-owned firm, the technology assumptions, the costs structure, and the hierarchy among the firms (in case of Stackelberg games). Another literature review that deals with some foundations for a theory of mixed oligopoly markets is presented in [14]. It is important to remark that all the above mentioned papers coincide in that there is a lack of an unified and accepted general mixed oligopolies modeling framework because each model pursues its own characteristics and set different basic hypothesis. At this point, it is also important to

${ }^{*}$ I am corresponding author

Email addresses: hmaravillo@hotmail.com (Héctor Maravillo), jose.camachovl@uanl.edu.mx (José-Fernando Camacho-Vallejo ), puerto@us.es (Justo Puerto), mlabbe@ulb.ac.be (Martine Labbé) 
emphasize that, to the best of our knowledge and after an intensive literature review, the intervention of the government via state-owned firms to regulate an industry conformed by two interrelated markets has not been studied before. However, these characteristics appear in real situations, as it will be described in the case study herein considered.

The problem studied in this paper is inspired by a situation where a state-owned firm controls the monopoly of a market but this is interrelated with a second market in which state-owned and private firms compete. The problem considers two interdependent industries, in which the first one produces the necessary supplies for the second one. The government has the monopoly of the first industry, while in the second industry a state-owned firm competes with remaining private firms in the production of commodities. Private firms aim to maximize their profit. Government aims as a general social welfare to balance the market. The latter is achieved by minimizing the difference between the supply and demand of the final commodities.

Given the privileged position that the government occupies in this market as the leader decision-maker, the government decides the amount of commodities that will be produced at the state-owned firms and the amount of supplies that will be offered to the private firms. By doing this, the government will regulate indirectly the production. One can easily appreciate a hierarchical relationship between both economic agents, in which the government makes a decision and the private firms reacts to it affecting the balance in the market. Furthermore, technical and technological issues in the raw material market limit the production, for example, the scarcity of natural resources and the existing production capacity of the firms. The hierarchy and inter-relationship among the actors of this situation allow to formulate a bilevel programming model, in which the government will act as the leader and the private firms as the follower. Other bilevel models in which the government intervenes to regulate social aspects are [15] and [16]. In the former, the government employs an intervention policy based on subsidies in the automotive market, while in the latter, the government apply taxes via an agro-environmental policy imposed to the agriculturists. Additionally, the general model proposed by us perfectly fits to the petrochemical industry in several developing countries in the last decades and it was the case of Mexico for almost 56 years, where it was apparently run without a theoretically recognizable model.

The main contributions of this paper can be listed as follows: a novel mathematical bilevel programming model to study a mixed-oligopoly market, three heuristic algorithms based on an iterative exploration of vertices in the inducible region of the bilevel problem, an extensive computational study analyzing the performance of our exact reformulations and the proposed heuristic algorithms, and a case-study based on the Mexican petrochemical industry.

The remainder of the paper is organized as follows. Section 2 defines the mathematical programming bilevel model and sets the notation. Two reformulations of the bilevel model that reduce it to a single-level one are presented in section 3. The first one is a continuous 
non-linear problem, and the second one reduces to a mixed integer linear program. In both cases, the resulting programs are hard to solve for medium to large sizes. Then, section 4 describes three proposed heuristic algorithms to provide good quality solutions for the bilevel model. Section 5 reports the numerical results according to the case study under consideration, and compares the results obtained through the reformulations and the algorithms. Also, the results obtained from additional experimentation with random instances are summarized. Conclusions and recommendations for future research are given in section 6 .

\section{A market regulation bilevel problem}

\subsection{Problem's description}

The problem herein studied considers an industry conformed by two economic markets, one of them associated to raw material and the other one of the final commodities. The supplies (raw material) of the second economic market are produced in the first one. In this industry, there is a state-owned firm vertically integrated, that is, that produces in both economic markets. The state-owned firm monopolizes the production of the supplies in the first economic market, but in the other one, this firm competes against the private firms. All the firms have a maximum production capacity and the state-owned firm requires to obtain a minimum profit (there is a lower bound over its net profit). The objective of this latter firm is to balance the market by its intervention. To achieve this goal, it minimizes the lack and surplus of the offered commodities with respect to the demand. Hence, this firm determines its production of commodities and the amount of supplies to be offered to the private firms. On the other hand, private firms' goal will be to maximize their benefit.

The decisions taken by the state-owned firm limit the admissible production by the private firms, and the decisions of the private firms affect the achievement of the government. As it is mentioned before, the government has the monopoly of the supplies production, and it is assumed that there exists a centralized organism that regulates the supplies demand. This organism determines the amount of supplies to each private firm, which is common in economic markets with government intervention. A typical example occurs when the government creates organisms to regulate the competition among firms and establishes particular contracts with each private firm fixing the supplies to be sold to each one. Another case is when the private firms get together and create a centralized mechanism to which the responsibility of distributing supplies among them is delegated. In the latter case, that mechanism seeks for a global welfare of the market; this is the case of common lands cooperative organization in agriculture. Under this scheme, the government will be the leader and the centralized mechanism the follower.

\subsection{Mathematical formulation}

In this section, the mathematical formulation of the problem described in the previous section is formally introduced. Let $I$ be the set of commodities and let $J$ be the set of private firms. Each commodity $i \in I$ is sold at a price $p_{i}$, and it has a demand $d_{i}$ in the 
market. To produce a commodity $i \in I$, it $\operatorname{costs} c_{i}^{G}$ to the state-owned firm and $c_{i j}^{E}$ to a private firm $j \in J$. The government fixes a minimum profit $t$ to be obtained by the stateowned firm. The amount of primary resources that a private firm $j \in J$ needs to produce a unit of commodity $i \in I$ is denoted by $b_{i j}$ and the amount of supply required to produce a unit of commodity $i \in I$ is denoted by $a_{i j}$. Both types of firms have a limited production capacity. The state-owned firm has a maximum production capacity $q_{i}^{B}$ to obtain supplies of the commodity $i \in I$ and a maximum amount $q_{i}^{A}$ to produce for each commodity $i \in I$. Also, each private firm $j \in J$ has a maximum production capacity $m_{j}$.

In order to present our mathematical programming formulation, we will use the following decision variables. The leader's decision variables are:

$x_{i}$, which denotes the production of the state-owned firm for each commodity $i \in I$.

$z_{i}$, which denotes the supply offered by the government to the private firms to produce a commodity in $\in I$.

The follower's decision variables are:

$y_{i j}$, which denotes the amount produced by private firm $j \in J$ of a commodity $i \in I$.

In our model, non-negative auxiliary variables are introduced to express the leader's objective function. Let $r_{i}$ be the shortage of commodity $i \in I$ and let $s_{i}$ be the corresponding surplus.

With the above elements, the proposed bilevel programming formulation to model the regulation of the economic market described above results as follows:

$$
\begin{aligned}
& \min _{x, z, r, s, y} \sum_{i \in I}\left(r_{i}+s_{i}\right) \\
& \text { s.t. } \frac{\sum_{j \in J} y_{i j}+x_{i}}{d_{i}}+r_{i}-s_{i}=1 \quad \forall i \in I \\
& \sum_{i \in I}\left(p_{i}-c_{i}^{G}\right) x_{i} \geq t \\
& 0 \leq x_{i} \leq q_{i}^{A} \quad \forall i \in I \\
& 0 \leq z_{i} \leq q_{i}^{B} \quad \forall i \in I \\
& r_{i} \geq 0 \quad \forall \in I i \\
& s_{i} \geq 0 \quad \forall i \in I \\
& y \quad \in \quad \operatorname{argmax} \sum_{j \in J} \sum_{i \in I}\left(p_{i}-c_{i j}^{E}\right) \bar{y}_{i j} \\
& \text { s.t. } \sum_{j \in J} a_{i j} \bar{y}_{i j} \leq z_{i} \quad \forall i \in I \\
& \sum_{i \in I} b_{i j} \bar{y}_{i j} \leq m_{j} \quad \forall j \in J
\end{aligned}
$$




$$
\bar{y}_{i j} \geq 0 \quad \forall i \in I, \quad \forall j \in J
$$

The leader's problem is defined by (1)-(8), in which (1) represents the leader's objective function that aims to minimize the inefficiency of the market, namely the overall shortage and surplus production with respect to the total demand. Constraint (2) seeks to balance the demand of each commodity with the corresponding production of the state-owned and private firms. In (3), it is ensured that the state-owned firm obtains a minimum required profit from its commodities production. Constraints (4) and (5) establish the production capacity associated with the commodities and offered supplies, respectively. The non-negativity of the auxiliary variables is expressed in (6) and (7). Constraint (8) plays a key role in this model, due to the fact that it requires that the value of the follower's variables is given by the optimal solution of another mathematical programming problem. The follower's problem is defined by equations (8)-(11), which aims to maximize the total profit of all the private firms. The production of the private firms is limited by the amount of supplies provided by the government, this is enforced by (9). Constraint (10) imposes that each private firm's production cannot exceed its maximum capacity. Finally, (11) expresses the non-negativity of the follower's variables.

In order to have a well-defined formulation for the proposed bilevel model, it is necessary to make an assumption regarding multiple optimal solutions that may appear in the follower's problem. We assume the optimistic approach defined in [17]. In other words, among all the optimal follower's solutions, the one that minimizes the leader's objective function is selected. The optimistic approach is suitable for the situation under study since it may express the existence of a certain cooperation degree among the government and the mechanism (committee) that represents the private firms.

\section{Exact solution methodologies}

A common methodology that applies, at times, to solve a bilevel programming model is to transform it to a single-level reformulation. In order to achieve this goal, the characteristics of the follower's problem have to be exploited. Once a leader's solution is established, the leader's variables are fixed in the follower's problem. Particularly, $z$ is going to be considered as a parameter in the problem defined by (8)-(11). Therefore, the follower's problem results in a linear programming problem that has a corresponding dual, in which $\alpha$ and $\beta$ correspond to the associated dual variables. The dual problem associated with the follower's problem is as follows:

$$
\begin{aligned}
& \min _{\alpha, \beta} \sum_{i \in I} \alpha_{i} z_{i}+\sum_{j \in J} \beta_{j} m_{j} \\
& \text { s.t. } \quad a_{i j} \alpha_{i}+b_{i j} \beta_{j} \geq\left(p_{i}-c_{i j}^{E}\right) \quad \forall i, \forall j \\
& \alpha_{i} \geq 0 \quad \forall i \\
& \beta_{j} \geq 0 \quad \forall j
\end{aligned}
$$


For both, the primal and dual of the follower's problem, the space of feasible solutions is bounded. Hence, the fundamental duality theorem states that both problems have optimal solutions and their objective function values are equal [18]. Hence, two single-level reformulations that are equal to the bilevel model are presented in this section. The first reformulation ensures that the optimal solution of the reformulated model are in the inducible region of the bilevel problem by using a corollary of the strong duality theorem. The second reformulation substitutes a non-linear constraint of the first reformulation via the complementarity slackness constraint. The first reformulation results in a continuous non-linear model and the second one is a mixed-integer linear model.

\subsection{Non-linear reformulation based on the strong duality condition}

The first reformulation consists in adding the constraints associated with the dual of the follower's problem, that is, (12-15), and to use the necessary and sufficient optimality conditions to ensure that the follower's decision belongs to the set of rational reactions. Therefore, a constraint that equals the follower's primal and dual objective functions is added. For a predefined leader's fixed value of $z$ the resulting constraint is as follows:

$$
\sum_{j \in J} \sum_{i \in I}\left(p_{i}-c_{i j}^{E}\right) y_{i j}=\sum_{i \in I} \alpha_{i} z_{i}+\sum_{j \in J} \beta_{j} m_{j}
$$

The first proposed reformulation consists in the following non-linear single-level problem:

$$
\begin{aligned}
& \min _{x, z, r, s, y, \alpha, \beta} \sum_{i \in I}\left(r_{i}+s_{i}\right) \\
& \text { s.t. } \begin{aligned}
& \frac{\sum_{j \in J} y_{i j}+x_{i}}{d_{i}}+r_{i}-s_{i}=1 \\
& \sum_{i \in I}\left(p_{i}-c_{i}^{G}\right) x_{i} \geq t
\end{aligned} \\
& 0 \leq x_{i} \leq q_{i}^{A} \quad \forall i \in I \\
& 0 \leq z_{i} \leq q_{i}^{B} \quad \forall i \in I \\
& r_{i} \geq 0 \quad \forall i \in I \\
& s_{i} \geq 0 \quad \forall i \in I \\
& \sum_{j \in J} a_{i j} y_{i j} \leq z_{i} \quad \forall i \in I \\
& \sum_{i \in I} b_{i j} y_{i j} \leq m_{j} \quad \forall j \in J \\
& y_{i j} \geq 0 \quad \forall i \in I, j \in J \\
& a_{i j} \alpha_{i}+b_{i j} \beta_{j} \geq\left(p_{i}-c_{i j}^{E}\right) \quad \forall i \in I, j \in J \\
& \alpha_{i} \geq 0 \quad \forall i \in I \\
& \beta_{j} \geq 0 \quad \forall j \in J
\end{aligned}
$$




$$
\sum_{j \in J} \sum_{i \in I}\left(p_{i}-c_{i j}^{E}\right) y_{i j}=\sum_{i \in I} \alpha_{i} z_{i}+\sum_{j \in J} \beta_{j} m_{j}
$$

Note that the linearity of the model is lost in (29). Further, it can be represented in the following manner since weak duality implies that the other inequality is always satisfied.

$$
-\sum_{j \in J} \sum_{i \in I}\left(p_{i}-c_{i j}^{E}\right) y_{i j}+\sum_{i \in I} \alpha_{i} z_{i}+\sum_{j \in J} \beta_{j} m_{j} \leq 0
$$

\subsection{Mixed-integer linear reformulation based on complementarity slackness}

The second reformulation is based on the complementarity slackness constraints that ensures the optimality of the follower's problem. A single-level reformulation is recovered by introducing the following constraints:

$$
\begin{aligned}
\alpha_{i}\left(z_{i}-\sum_{j \in J} a_{i j} y_{i j}\right) & =0 & & \forall i \in I \\
\beta_{j}\left(m_{j}-\sum_{i \in I} b_{i j} y_{i j}\right) & =0 & & \forall j \in J \\
y_{i j}\left(a_{i j} \alpha_{i}+b_{i j} \beta_{j}-\left(p_{i}-c_{i j}^{E}\right)\right) & =0 & & \forall i \in I, j \in J
\end{aligned}
$$

Constraints (31), (32), and (33) are non-linear, nevertheless they can be linearized in a straightforward manner by introducing positive big- $M$ constants $M_{i}^{1}, M_{i}^{2}$, and $M_{i}^{3}$ for all $i \in I$. Also, binary variables $\gamma_{i}, \delta_{j}$, and $\epsilon_{i j}$ for all $i \in I, j \in J$ are included. Constraints (31)-(33) are replaced by the following constraints:

$$
\begin{aligned}
\alpha_{i} & \leq M_{i}^{1} \gamma_{i} & & \forall i \in I \\
z_{i}-\sum_{j \in J} a_{i j} y_{i j} & \leq M_{i}^{1}\left(1-\gamma_{i}\right) & & \forall i \in I \\
\beta_{j} & \leq M_{i}^{2} \delta_{j} & & \forall i \in I, j \in J \\
m_{j}-\sum_{i \in I} b_{i j} y_{i j} & \leq M_{i}^{2}\left(1-\delta_{j}\right) & & \forall i \in I, j \in J \\
y_{i j} & \leq M_{i}^{3} \epsilon_{i j} & & \forall i \in I, \forall j \in J \\
a_{i j} \alpha_{i}+b_{i j} \beta_{j}-\left(p_{i}-c_{i j}^{E}\right) & \leq M_{i}^{3}\left(1-\epsilon_{i j}\right) & & \forall i \in I, j \in J
\end{aligned}
$$

Therefore, this reformulation results in the following single-level linear mixed-integer programming model.

$$
\begin{aligned}
\min _{x, z, r, s, y, \alpha, \beta, \gamma, \delta, \epsilon} & \sum_{i \in I}\left(r_{i}+s_{i}\right) \\
\text { s.t. } & \frac{\sum_{j \in J} y_{i j}+x_{i}}{d_{i}}+r_{i}-s_{i}=1 \quad \forall i \in I
\end{aligned}
$$




$$
\begin{aligned}
\sum_{i \in I}\left(p_{i}-c_{i}^{G}\right) x_{i} & \geq t \\
0 \leq x_{i} & \leq q_{i}^{A} \quad \forall i \in I \\
0 \leq z_{i} & \leq q_{i}^{B} \quad \forall i \in I \\
r_{i} & \geq 0 \quad \forall i \in I \\
s_{i} & \geq 0 \quad \forall i \in I \\
\sum_{j \in J} a_{i j} y_{i j} & \leq z_{i} \quad \forall i \in I \\
\sum_{i \in I} b_{i j} y_{i j} & \leq m_{j} \quad \forall j \in J \\
y_{i j} & \geq 0 \quad \forall i, j \in J \\
a_{i j} \alpha_{i}+b_{i j} \beta_{j} & \geq\left(p_{i}-c_{i j}^{E}\right) \quad \forall i \in I, j \in J \\
\alpha_{i} & \geq 0 \quad \forall i \in I \\
\beta_{j} & \geq 0 \quad \forall j \in J \\
\alpha_{i} & \leq M_{i}^{1} \gamma_{i} \quad \forall i \in I \\
z_{i}-\sum_{j \in J} a_{i j} y_{i j} & \leq M_{i}^{2}\left(1-\gamma_{i}\right) \quad \forall i \in I \\
\beta_{j} & \leq M_{j}^{1} \delta_{j} \quad \forall j \in J \\
\alpha_{i}, \delta_{j}, \epsilon_{i j} & \in\{0,1\} \quad \forall i \in I, j \in J \\
b_{i j} \beta_{j}-\left(p_{i}-c_{i j}^{E}\right) & \leq M_{i j}^{2}\left(1-\epsilon_{i j}\right) \quad \forall i \in I, j \in J \\
y_{i \in I} b_{i j} y_{i j} & \leq M_{j}^{2}\left(1-\delta_{j}\right) \quad \forall j \in J \\
&
\end{aligned}
$$

\subsection{Adjusting the value of the big $M$ 's}

In the problem under study, parameters $a$ and $b$ are always positives, which means that if more commodity production exists, more supplies are required and more production capacity is used in the private firms. Moreover, if term $p_{i}-c_{i j}^{E}$ is negative, private firms are having losses, which would lead to their exit from the economic market.

Definition. Define the upper bound $U B(\cdot) \in \mathbb{R}$ of a vector as a real number that is is greater than or equal to all of the components of that vector.

To adjust the value of $M_{i}^{1}$ for all $i \in I$, first we seek for an upper bound for $\alpha_{i}$, such that $\alpha$ belongs to an optimal solution of the follower's dual problem. The worst scenario occurs when $\beta_{j}=0 \forall j \in J$, then $\alpha_{i} \geq \frac{p_{i}-c_{i j}^{E}}{a_{i j}}$ for all $i \in I, \forall j \in J$ must hold due to (49). Since (12) aims to minimize, the optimal solution occurs at equality. To obtain the upper bound for $M_{i}^{2}$ for all $i \in I$, constraint (53) is considered. Hence, it is possible to state the following proposition. 


\section{Proposition 1.}

1. The upper bound for any optimal value of $\alpha_{i}$ for all $i \in I$ is as follows:

$$
U B\left(\alpha_{i}\right)=\max _{j \in J}\left\{\frac{p_{i}-c_{i j}^{E}}{a_{i j}}\right\}
$$

2. The upper bound for constraint (53) is as follows:

$$
U B\left(z_{i}-\sum_{j \in J} a_{i j} y_{i j}\right)=q_{i}^{B} .
$$

Analogously that for $M_{i}^{k}$, the upper bounds for $M_{j}^{k} i=1,2$ and $k=1,2$ are computed.

\section{Proposition 2.}

1. The upper bound for any optimal value of $\beta_{j}$ for all $j \in J$ is:

$$
U B\left(\beta_{j}\right)=\max _{i \in I}\left\{\frac{p_{i}-c_{i j}^{E}}{b_{i j}}\right\} .
$$

2. The upper bound for constraint (55) is:

$$
U B\left(m_{j}-\sum_{i \in I} b_{i j} y_{i j}\right)=m_{j} .
$$

To bound the values of $M_{i j}^{k}$ for all $i \in I, j \in J$ and $k=1,2$, the following proposition is stated.

\section{Proposition 3.}

1. The upper bounds for $y_{i j}$ for all $i \in I, j \in J$ are:

$$
U B\left(y_{i j}\right)=\frac{m_{j}}{b_{i j}}
$$

i.e. $y_{i j} \leq \frac{m_{j}}{b_{i j}}$ for all $i \in I, j \in J$.

2. The upper bound of constraint (57) is:

$$
U B\left(a_{i j} \alpha_{i}+b_{i j} \beta_{j}-\left(p_{i}-c_{i j}^{E}\right)\right)=a_{i j} U B\left(\alpha_{i}\right)+b_{i j} U B\left(\beta_{j}\right) .
$$

Therefore, the adjusted values for $M_{i}^{k}, M_{j}^{k}$, and $M_{i j}^{k}$ for all $i \in I, j \in J, k=1,2$ based on the computed upper bounds are as follows:

$$
\begin{aligned}
M_{i}^{1} & =\max _{j \in J}\left\{\frac{p_{i}-c_{i j}^{E}}{a_{i j}}\right\}, \quad \forall i \in I \\
M_{i}^{2} & =q_{i}^{B}, \quad \forall i \in I \\
M_{j}^{1} & =\max _{i \in I}\left\{\frac{p_{i}-c_{i j}^{E}}{b_{i j}}\right\}, \quad \forall j \in J \\
M_{j}^{2} & =m_{j}, \quad \forall j \in J \\
M_{i j}^{1} & =\frac{m_{j}}{b_{i j}}, \quad \forall i \in I, j \in J \\
M_{i j}^{2} & =a_{i j} U B\left(\alpha_{i}\right)+b_{i j} U B\left(\beta_{j}\right), \quad \forall i \in I, j \in J .
\end{aligned}
$$




\section{Heuristic solution methodologies}

Usually, the reformulations introduced above present computational limitations for largesize instances. Therefore, alternative approaches are required to solve the bilevel problem. One common approach is to design a heuristic algorithm to obtain good quality feasible solutions with lower computational burden. In this section, three tailor-made heuristic algorithms are proposed to solve the problem under study. The first two heuristics exploit the particular structure of the bilevel problem, while the third one hybridizes the other two. The hybrid heuristic contains key aspects from the previously proposed heuristics to enhance its performance.

Note that it has been already emphasized that the polyhedron that defines the feasible region of the dual problem associated with the follower is independent from the leader's variables. Therefore, if all the vertices of this polyhedron were known, equality (16) and the dual constraints of the first reformulation described in section 3.1 could be substituted by a set of constraints guaranteeing that the equality (16) would be achieved in one vertex. Following the latter idea, the dual variables are replaced by parameters that represents those known vertices. This is the approach exploited in the three proposed heuristic algorithms.

\subsection{Extreme points iterated algorithm (EPIA)}

In this algorithm, vertices of the dual polyhedron are iteratively generated, and a mixedinteger programming problem named the Master Problem (MP) is solved for each new vertex. The optimal solution of the MP ensures a feasible solution of the bilevel problem. The algorithm stops when no improvement in the leader's objective function is obtained.

Let $P$ be the polyhedron associated with the dual constraints of problem (13)-(15). Consider $v_{k}=\left(\alpha^{k}, \beta^{k}\right) \in P, k=1, \ldots,|P|$ as the vertices of $P$, in which $|P|$ define the number of vertices in that polyhedron with $\alpha^{k}=\left(\alpha_{1}^{k}, \ldots, \alpha_{|I|}^{k}\right)$ and $\beta^{k}=\left(\beta_{1}^{k}, \ldots, \alpha_{|J|}^{k}\right)$. Let $F$ be the leader's objective function (1). Also, let $f$ and $g$ be the follower primal (8) and dual (12) objective functions, respectively. Consider $\chi=(x, z, r, s)$ as a vector that groups the leader's variables and $\Upsilon=\left(y_{1}, \ldots, y_{|J|}\right)$ as a vector that groups the follower's variables. Define $\varphi$ as the set of vertices in $P$ that has been already explored. At each iteration $K=|\varphi|$ is updated using $k \in K$ as the index of the explored vertices. $U B\left(z_{i}\right)$ represents an upper bound of variable $z_{i}$, which can be naturally fixed as $q_{i}^{B}$. Hence, the MP is defined as follows:

$$
\begin{array}{rlrl}
\min _{x, z, r, s, y, \lambda} \sum_{i \in I}\left(r_{i}+s_{i}\right) & & & \\
\text { s.t. } \frac{\sum_{j \in J} y_{i j}+x_{i}}{d_{i}+r_{i}-s_{i}} & =1 & & \forall i \in I \\
\sum_{i \in I}\left(p_{i}-c_{i}^{G}\right) x_{i} & \geq t & \\
0 \leq x_{i} & \leq q_{i}^{A} & & \forall i \in I \\
0 \leq z_{i} & \leq q_{i}^{B} & & \forall i \in I
\end{array}
$$




$$
\begin{array}{rlrl}
r_{i} & \geq 0 & \forall i \in I \\
s_{i} & \geq 0 & \forall i \in I \\
\sum_{j \in J} a_{i j} y_{i j} & \leq z_{i} & \forall i \in I \\
\sum_{i \in I} b_{i j} y_{i j} & \leq m_{j} & \forall j \in J \\
y_{i j} & \geq 0 & \forall i \in I, j \in J \\
\sum_{i \in I} \alpha_{i}^{k} z_{i}+\sum_{j \in J} \beta_{j}^{k} m_{j}-\left(1-\lambda_{k}\right)\left(\sum_{i \in I} \alpha_{i}^{k} U B\left(z_{i}\right)+\sum_{j \in J} \beta_{j}^{k} m_{j}\right) & \leq \sum_{j \in J} \sum_{i \in I}\left(p_{i}-c_{i j}^{E}\right) y_{i j} \forall k \in K \\
\sum_{k \in K} \lambda_{k} & =1 & \\
\lambda_{k} & \in\{0,1\} & \forall k \in K
\end{array}
$$

Constraints (75)-(77) guarantee that $f(\Upsilon)=g\left(\chi, v_{k}\right)$ holds only for a single vertex, in which $\lambda$ is an auxiliary binary variable. The obtained solution $\Upsilon^{*}$ belongs to the rational reaction set. Hence, the optimal solution of MP is a feasible solution of the bilevel problem.

The process in which the dual problem (12)-(15) is solved for a fixed vector $\mathbf{z}$ obtaining an optimal solution $v=(\alpha, \beta)$ is represented as $D(z) \rightarrow v$. Analogously, solve MP for a set of vertices $\varphi$ is denoted as $\operatorname{MP}(\varphi) \rightarrow(\chi, \Upsilon)$. The result is a pair of solutions $(\chi, \Upsilon)$. The pseudocode of the EPIA is presented in algorithm 4.1.

Algorithm 4.1 Extreme points iterated algorithm

Step 1. Initialization: $\varphi=\emptyset, \rho=\infty$;

Step 2. Find the initial vertices $D(\mathbf{0}) \rightarrow v_{1} . D\left(q_{i}^{B}\right) \rightarrow v_{2} . \varphi=\varphi \cup\left\{v_{1}, v_{2}\right\}$;

Step 3. Solve the master problem: $\operatorname{MP}(\varphi) \rightarrow\left(\chi^{k}, \Upsilon^{k}\right) . \pi=F\left(\chi^{k}, \Upsilon^{k}\right)$;

Step 4. Find a vertex $k: D\left(z^{k}\right) \rightarrow v_{k}$;

Step 5. Evaluate leader's objective function.

- If $\rho>\pi$ then $\varphi=\varphi \cup\left\{v_{k}\right\}, \rho=\pi$. Return to Step 3;

- If $\rho \leq \pi$ then stops.

Output: $\pi,\left(\chi^{k}, \Upsilon^{k}\right)$

Note that optimality of the bilevel problem is not guaranteed by this algorithm. The main reason is that during the process, the algorithm may not improve the leader's objective function. This may occurs when the value $z^{k}$ obtained at iteration $k$ produces a vertex $v_{k}$ that is already in set $\varphi$, or simply when $F\left(\chi^{k}, \Upsilon^{k}\right) \geq F\left(\chi^{k-1}, \Upsilon^{k-1}\right)$.

\subsection{Follower's gap penalization algorithm (FGPA)}

The general idea of FGPA is to find a feasible solution by using vertices of the follower's dual problem, but the dual admits infeasible solutions. The algorithm consists on itera- 
tively solving a mixed-integer programming problem, named MMP, that is a modification of the MP described in previous section. The MMP permits the existence of a gap between follower's primal objective function and the dual objective function value obtained by evaluating the considered vertices. However, this gap is penalized in the leader's objective function aiming to obtain a good feasible solution.

Since the primal and dual objective values of the follower can be different, the algorithm may explore other dual vertices using solutions that are in the constraint region of the bilevel problem, but not necessarily in the inducible region. Hence, to ensure feasibility, at the end of the iterations another problem named Resulting problem (RP) must be solved. RP is a linear single-level problem defined by constraints (1)-(7) of the leader, constraints (9)-(11) of the follower, and a constraint that equals $f(\Upsilon)$ with the value of the dual objective function $g$ evaluated with the last obtained value of $\mathbf{z}\left(\hat{z}=z^{k}\right)$. Therefore, $\psi=g\left(z^{k}\right)$ is a parameter used in:

$$
\sum_{j \in J} \sum_{i \in I}\left(p_{i}-c_{i j}^{E}\right) y_{i j}=\psi
$$

In MMP, the gap is normalized by a constant $M$ and multiplied by a coefficient $\mu$ to regulate the impact in the leader's objective function, that is, to balance the supply and demand. The value of $M$ can be bounded by the maximum among all the optimal values of the dual problem obtained at each iteration. The modified master problem (MMP) is follows:

$$
\begin{array}{rlrl}
\min _{x, z, r, s, y, \lambda, \varepsilon} \sum_{i \in I}\left(r_{i}+s_{i}\right)+\frac{\varepsilon}{M} \mu & & \\
\text { s.t. } \frac{\sum_{j \in J} y_{i j}+x_{i}}{d_{i}+r_{i}-s_{i}} & =1 & & \\
\sum_{i \in I}\left(p_{i}-c_{i}^{G}\right) x_{i} & \geq t & & \forall i \in I \\
0 \leq x_{i} & \leq q_{i}^{A} & & \\
0 \leq z_{i} & \leq q_{i}^{B} & & \forall i \in I \\
r_{i} & \geq 0 & & \forall i \in I \\
s_{i} & \geq 0 & & \forall i \in I \\
\sum_{j \in J} a_{i j} y_{i j} & \leq z_{i} & & \forall i \in I \\
\sum_{i \in I} b_{i j} y_{i j} & \leq m_{j} & & \forall i \in I \\
y_{i j} & \geq 0 & & \forall j \in J \\
\sum \lambda_{k} & =1 & & \forall i \in I, j \in J \\
\lambda_{k} & \in\{0,1\} & & \forall k \in K
\end{array}
$$




$$
\begin{aligned}
\varepsilon^{k}+\sum_{j \in J} \sum_{i \in I}\left(p_{i}-c_{i j}^{E}\right) y_{i j} & =\sum_{i \in I} \alpha_{i}^{k} z_{i}+\sum_{j \in J} \beta_{j}^{k} m_{j} \quad \forall k \in K \\
\varepsilon^{k} & \geq 0 \quad \forall k \in K \\
\varepsilon & \geq 0 \\
\varepsilon & \geq \varepsilon^{k}-\left(1-\lambda_{k}\right) M \quad \forall k \in K
\end{aligned}
$$

Therefore, the RP that is used to obtain a solution in the inducible region is:

$$
\begin{aligned}
\min _{x, r, s, y} \sum_{i \in I}\left(r_{i}+s_{i}\right) & & & \\
\text { s.t. } \frac{\sum_{j \in J} y_{i j}+x_{i}}{d_{i}+r_{i}-s_{i}} & =1 & & \\
\sum_{i \in I}\left(p_{i}-c_{i}^{G}\right) x_{i} & \geq t & & \\
0 \leq x_{i} & \leq q_{i}^{A} & & \\
r_{i} & \geq 0 & & \forall i \in I \\
s_{i} & \geq 0 & & \forall i \in I \\
\sum_{j \in J} a_{i j} y_{i j} & \leq \hat{z}_{i} & & \forall i \in I \\
\sum_{i \in I} b_{i j} y_{i j} & \leq m_{j} & & \forall i \in I \\
y_{i j} & \geq 0 & & \forall j \in J \\
\sum_{j \in J} \sum_{i \in I}\left(p_{i}-c_{i j}^{E}\right) y_{i j} & =\psi & & \forall i, j \in J
\end{aligned}
$$

To refer to the $\operatorname{MMP}$, define $\operatorname{MMP}(\varphi, M) \rightarrow(\chi, \Upsilon)$ as in the previous algorithm. The leader's objective function (79) is represented by $F^{*}$. Also, $\operatorname{RP}(\varphi, \psi, \hat{z}) \rightarrow(\chi, \Upsilon)$ denotes the process of solving RP using the set of vectors $\varphi$ and the parameters $\psi$ and $\hat{z}$. The pseudocode of FGPA is shonw in algorithm 4.2.

\subsection{Hybrid algorithm (HYBA)}

As mentioned before, the EPIA cannot ensure to obtain a new vertex in each iteration nor a vertex that improves the leader's objective function. An intuitive idea is to use the FGPA to avoid this issue. The latter algorithm has de advantage of exploring solutions that does not belong to the inducible region, by doing this, unexplored vertices are obtained.

The main idea of the HYBA is to perform the steps of EPIA until stops. When this occurs, a subroutine that solves the MMP is performed to find a new vertex aiming to reach a different feasible solution. If the new exploration improves the leader's objective function, 
Algorithm 4.2 Follower's gap penalization algorithm

Step 1. Initialization: $\varphi=\emptyset, \rho=\infty, M=\operatorname{Max}\left\{1, D\left(q_{i}^{B}\right)\right\}$;

Step 2. Solve the modified master problem: $\operatorname{PMM}(\varphi, M) \rightarrow\left(\chi^{k}, \Upsilon^{k}\right) . \pi=F^{*}\left(\chi^{k}, \Upsilon^{k}\right)$;

Step 3. Find a vertex k: $D\left(z^{k}\right) \rightarrow v_{k} . M=\operatorname{Max}\left\{M, D\left(z^{k}\right)\right\}$;

Step 4. Evaluate leader's objective funcion.

- If $\rho>\pi$ then $\varphi=\varphi \cup\left\{v_{k}\right\}, \rho=\pi$. Return to Step 2;

- If $\rho \leq \pi$ then go to Step 5 .

Step 5. Obtain a feasible solution: $\psi=D\left(z_{k}\right) \cdot \operatorname{RP}(\varphi, \psi, \hat{z}) \rightarrow\left(\chi^{*}, \Upsilon^{*}\right) . \pi=F\left(\chi^{*}, \Upsilon^{*}\right)$;

Output: $\pi,\left(\chi^{*}, \Upsilon^{*}\right)$

then the FGPA continues until the stopping criterion is reached. The pseudocode of the proposed hybrid algorithm is shown in algorithm 4.3.

Algorithm 4.3 Hybrid algorithm

Step 1. Initialization: $\varphi=\emptyset, \rho=\infty$;

Step 2. Find the initial vertices: $D(\mathbf{0}) \rightarrow v_{1} . \quad D\left(q_{i}^{B}\right) \rightarrow v_{2} . \varphi=\varphi \cup\left\{v_{1}, v_{2}\right\}, \quad M=$ $\operatorname{Max}\left\{D(\mathbf{0}), D\left(q_{i}^{B}\right)\right\}$;

Step 3. Solve the master problem: $\operatorname{MP}(\varphi) \rightarrow\left(\chi^{k}, \Upsilon^{k}\right) \cdot \pi=F\left(\chi^{k}, \Upsilon^{k}\right)$;

Step 4. Find a vertex $k: D\left(z^{k}\right) \rightarrow v_{k} . \varphi=\varphi \cup\left\{v_{k}\right\}, M=\operatorname{Max}\left\{M, D\left(z^{k}\right)\right\}$;

Step 5. Evaluate leader's objective function.

- If $\rho>\pi$ then Go to Step 6;

- If $\rho \leq \pi$ then

$\operatorname{MMP}(\varphi, M) \rightarrow\left(\chi^{k^{*}}, \Upsilon^{k^{*}}\right) . D\left(z^{k^{*}}\right) \rightarrow v_{k^{*}} \cdot \varphi=\varphi \cup\left\{v_{k^{*}}\right\}$

$\operatorname{MP}(\varphi) \rightarrow\left(\chi^{k}, \Upsilon^{k}\right) . \quad \pi=F\left(\chi^{k}, \Upsilon^{k}\right) . D\left(z^{k}\right) \rightarrow v_{k}, \varphi=\varphi \cup\left\{v_{k}\right\}, \quad M=$ $\operatorname{Max}\left\{M, D\left(z^{k}\right)\right\}$.

Step 6. Re-evaluating leader's objective function:

- If $\rho>\pi$ then $\rho=\pi$. Return to Step 3;

- If $\rho \leq \pi$ then Stops.

Output: $\pi,\left(\chi^{k}, \Upsilon^{k}\right)$

\section{Computational experimentation}

In this section, the results obtained from an extensive computational experimentation of our solution methodologies are reported. To evaluate both single-level reformulations and 
the performance of the proposed heuristic algorithms, a set of 360 instances was used. The first subset of 180 instances corresponds to a case based on a real situation occurred in the Mexican petrochemical industry between 1958-2014. The second subset of 180 instances was randomly generated to test the efficiency of our algorithms in more general data sets.

All the computational experiments were conducted in a personal computer Dell Inspiron 5558, with the following characteristics: a processor Intel(R) Core i3 with $2.10 \mathrm{GHz}, 6.00$ GB of RAM under Windows 10 operative system. The mixed-integer linear reformulation based on the complementarity slackness condition (hereafter Ref.2), and the three proposed heuristic algorithms were implemented in C++ using Microsoft Visual Studio 2017. The optimizer used was CPLEX 12.7.1. On the other hand, the non-linear reformulation based on the strong duality condition (hereafter Ref.1) was implemented in AMPL using Baron 18.5.8 as optimizer.

\subsection{Case study}

In Mexico, the government intervention in the petrochemical industry existed by many decades. The main characteristic was that the government monopolized the extraction of the main raw material for this industry, namely petroleum and some other derivatives called basic petrochemicals. Another characteristic of this situation was that state-owned firms competed against private firms in the market of final commodities (secondary petrochemicals).

The institutional framework defined by law the petrochemical industry as the one that performs chemical or physical processes to product compounds from petroleum natural hydrocarbons or from the products derived from refinement operations. Some of these products may serve as raw materials to the industry, and they were classified as part of the basic petrochemicals. The remaining products were included into the secondary petrochemical category [19].

Specifically, in a law from 1958 [20], it was established that only the government was allowed to exploit the hydrocarbons related with the oil industry, which was concerned with the production, warehousing, distribution, and sales of the petroleum derivatives that can be considered as raw materials (basic petrochemicals) for the industry. However, for the production of secondary petrochemicals products, state-owned and private firms were allowed to be involved in. Therefore, Petróleos Mexicanos (PEMEX) and its subsidiaries was in charge of this industry [21], and from 1992, also their descentralized departments [22]. The classification of basic and secondary petrochemical products varies by law from year to year [23], [21], [19], [24], [25], [26], [27].

To delimit the scope and size of our case study instances based on the above situation, we consider the number of economic units involved in the manufacturing of organic chemical basic products registered by the National Institute of Statistics and Geography in Mexico (INEGI by its acronym in Spanish) during the economical census conducted in 1999, 2004, 
2009, and 2014. The biggest number of these units was registered in 2004, which was 159. This value is used as an upper bound of the private firms dedicated to secondary petrochemical industry. For delimiting the amount of commodities considered, we consider the number of commodities classified as basic and secondary petrochemical by the Mexican legislation in different years. This information is summarized in table 1.

Table 1: Classification of petrochemical commodities

\begin{tabular}{lrr}
\hline $\begin{array}{c}\text { Year of the } \\
\text { classification }\end{array}$ & $\begin{array}{c}\text { Basic } \\
\text { petrochemicals }\end{array}$ & $\begin{array}{c}\text { Secondary } \\
\text { petrochemicals }\end{array}$ \\
\hline 1960 & 16 & - \\
1986 & 34 & 26 \\
1989 & 8 & 13 \\
1992 & 20 & 35 \\
\hline Obtained by using data from [21], [24], [25], [26]
\end{tabular}

To complete the instances, we extracted data from the statistical report of the energy sector used by the Mexican Secretary of Energy. The data of 47 petrochemical commodities from 1980 to 2002 were obtained. The information consists of the demand, prices, and productive capacity. For each commodity $i$, the demand $d_{i}$ was generated from a uniform distribution with the following parameters: the average of the demands in that period of time minus the standard deviation, and the average of the demands in that period of time plus the standard deviation. The price $p_{i}$ and the government production capacity $q_{i}^{A}$ were randomly generated between 1 and the maximum price or the maximum capacity for each commodity $i$, respectively.

The government $\operatorname{costs} c_{i}^{G}$ were obtained as the product of the price of each commodity $i$ times a random number between 0.22 and 0.60 . The latter range was defined based on the ratio between the production costs and the PEMEX (the Mexican state-owned firm) total income registered from the years 1988-2000, 2011-2013, and 2015-2016. The minimum profit that the state-owned firm must achieved was computed as the $30 \%$ of the total benefit that the government may obtain if all the petrochemicals market demand was satisfied by PEMEX.

The state-owned firm has a production capacity $q_{i}^{B}$ regarding raw materials $i$, which was computed as the product of the maximum production coefficient of the private firms and the maximum capacity of the state-owned firm for producing commodities. Under this assumption, the private firm with best technology could match the production capacity of the state-owned firm for an specific commodity.

Production $\operatorname{costs} c_{i j}^{E}$ of private firm $j$ are defined as the product of random coefficients in $[0.784,0.884]$ multiplied times the price of each commodity $i$. That range corresponds to 
the minimum and maximum average values of the ration between the production costs and the income per year of five real private firms. These private firms operate in the Mexican petrochemical sector: Alpek, Kuo (UEN synthetic rubber), Kuo (UEN polystyrene), Mexichem, and Pochteca group.

The production technical coefficients $a_{i j}$ are randomly generated between [0.085, 2.111], which correspond to the range determined by the average plus/minus one standard deviation of all the petrochemical substances considered in the report presented by CEPAL [? ]. The production levels $b_{i j}$ are generated from the interval $[1,95]$. The lower bound is natural since these coefficients must be strictly positives, while the upper bound is equal to the estimated average of the production levels for each substance considered in the petrochemical facilities of PEMEX.

Finally, the production capacity $m_{j}$ of the private firms were randomly generated between 4,665 and 20,825, which came from the average of the production capacity of five petrochemical complex of PEMEX.

Based on the above factors and ranges, we generated 30 instances for each one of the following sizes:

- $|I|=10,|J|=10$

- $|I|=25,|J|=25$

- $|I|=25,|J|=75$

- $|I|=50,|J|=100$

- $|I|=75,|J|=125$

- $|I|=150,|J|=200$

As it is mentioned above, the instances generated to analyze the case study were taken as a basis for constructing another set of synthetic instances to test the performance of our algorithms on a different data set. This new set of instances has the same sizes but were randomly generated using arbitrary ranges for each parameter. Furthermore, the generation scheme guarantees feasibility of the problem, that is, ensure that the state-owned firm has capacity to produce all the demand.

\subsection{Numerical results}

Our computational experiment consists on solving both subsets of instances described above, that is, the case-study and the synthetic instances. All of them were solved by the two reformulations presented in section 3 and by the three heuristic algorithms proposed in section 4 . The leader's objective function value and the required time were registered. Among the two exact methods, only Ref.2 was able to optimally solve all the tested instances. 
Hence, these values were used to compute the optimality gap obtained by the heuristic algorithms. A maximum CPU time of 1000 seconds was set to the solver for solving Ref.1, while Ref.2 and the heuristic algorithms we did not fix a time limit since in all cases the required time was rather small.

We observe from our results, that Ref.1 was not able to solve all the instances within the time limit. We also observe that the case-study instances are harder to be solved than the synthetic ones. For example, for the larger instance sizes $(150 \times 200)$, Ref.1 did not even find feasible solutions for the problem. This may be due to the fact that synthetic instances were well-balanced so that, in most cases, shortages and surplus are both zero. Summarizing, the number of instances solved to optimality by each reformulation is shown in table 2 .

Table 2: Number of instances solved to optimality

\begin{tabular}{clrr}
\hline Instances & \multicolumn{1}{c}{ Size } & Ref.1 & Ref.2 \\
\hline & $10 \times 10$ & 15 & 30 \\
& $25 \times 25$ & 0 & 30 \\
Case-study & $25 \times 75$ & 0 & 30 \\
& $50 \times 100$ & 0 & 30 \\
& $75 \times 125$ & 0 & 30 \\
& $150 \times 200$ & 0 & 30 \\
& $10 \times 10$ & 30 & 30 \\
Synthetic & $25 \times 25$ & 29 & 30 \\
& $50 \times 100$ & 29 & 30 \\
& $75 \times 125$ & 28 & 30 \\
& $150 \times 200$ & 13 & 30 \\
\hline
\end{tabular}

The following tables 3 and 4 report all the results of our experiment. The results are organized in five column blocks corresponding to the five solution methods that are compared. In each block, we include two columns with the objective function values $(\mathrm{F})$ and the $\mathrm{CPU}$ time (t). In these tables, averages of the registered values for each size of the case-study and synthetic instances are shown. It is worth mentioning that when Ref.1 was not able to solve the instance, that instance was omitted for the computation of the corresponding average value. 
Table 3: Summarized results obtained from the case-study instances

\begin{tabular}{lrrrrrrrrrr}
\hline \multicolumn{1}{c}{ Size } & \multicolumn{2}{c}{ Ref.1 } & \multicolumn{2}{c}{ Ref.2 } & \multicolumn{2}{c}{ EPIA } & \multicolumn{2}{c}{ FGPA } & \multicolumn{2}{c}{ HYBA } \\
& $\overline{\mathrm{F}}$ & $\overline{\mathrm{t}}(\mathrm{s})$ & $\overline{\mathrm{F}}$ & $\overline{\mathrm{t}}(\mathrm{s})$ & $\overline{\mathrm{F}}$ & $\overline{\mathrm{t}}(\mathrm{s})$ & $\overline{\mathrm{F}}$ & $\overline{\mathrm{t}}(\mathrm{s})$ & $\overline{\mathrm{F}}$ & $\overline{\mathrm{t}}(\mathrm{s})$ \\
\hline $10 \times 10$ & 0.561 & 255.56 & 0.506 & 0.18 & 0.774 & 0.23 & 0.825 & 0.16 & 0.524 & 0.41 \\
$25 \times 25$ & 14.225 & 500.26 & 1.000 & 1.86 & 1.570 & 0.30 & 1.171 & 0.35 & 1.020 & 0.71 \\
$25 \times 75$ & 12.435 & 500.46 & 0.527 & 4.36 & 1.025 & 0.48 & 0.565 & 0.39 & 0.552 & 1.02 \\
$50 \times 100$ & 211.696 & 558.80 & 1.051 & 53.15 & 2.041 & 2.39 & 1.132 & 1.14 & 1.091 & 5.18 \\
$75 \times 125$ & 431.138 & 501.24 & 1.546 & 268.89 & 2.339 & 8.89 & 1.811 & 3.68 & 1.621 & 20.69 \\
$150 \times 200$ & - & - & 3.279 & 2754.68 & 4.129 & 61.82 & 5.037 & 12.06 & 3.443 & 112.25 \\
\hline
\end{tabular}

Table 4: Summarized results obtained from the synthetic instances

\begin{tabular}{lrrrrrrrrrrr}
\hline \multicolumn{1}{c}{ Size } & \multicolumn{2}{c}{ Ref.1 } & \multicolumn{2}{c}{ Ref.2 } & \multicolumn{2}{c}{ EPIA } & \multicolumn{2}{c}{ FGPA } & \multicolumn{2}{c}{ HYBA } \\
& $\overline{\bar{F}}$ & $\overline{\mathrm{t}}(\mathrm{s})$ & $\overline{\mathrm{F}}$ & $\overline{\mathrm{t}}(\mathrm{s})$ & $\overline{\mathrm{F}}$ & $\overline{\mathrm{t}}(\mathrm{s})$ & $\overline{\mathrm{F}}$ & $\overline{\mathrm{t}}(\mathrm{s})$ & $\overline{\mathrm{F}}$ & $\overline{\mathrm{t}}(\mathrm{s})$ \\
\hline $10 \times 10$ & 0 & 0.26 & 0 & 0.14 & 0.007 & 0.08 & 0.006 & 0.10 & 0 & 0.09 \\
$25 \times 25$ & 0.012 & 29.01 & 0 & 1.43 & 0 & 0.11 & 0.021 & 0.14 & 0 & 0.11 \\
$25 \times 75$ & 0 & 10.94 & 0 & 3.65 & 0 & 0.17 & 0.019 & 0.20 & 0 & 0.17 \\
$50 \times 100$ & 0.057 & 73.60 & 0 & 35.52 & 0 & 0.29 & 0.170 & 0.31 & 0 & 0.28 \\
$75 \times 125$ & 0 & 118.76 & 0 & 90.48 & 0 & 0.60 & 0.086 & 0.76 & 0 & 0.57 \\
$150 \times 200$ & 1.657 & 414.55 & 0 & 193.48 & 0 & 1.78 & 0.443 & 1.74 & 0 & 1.57 \\
\hline
\end{tabular}

We observe from tables 3 and 4 that Ref.1 (recall that Ref.1 is a continuous non-linear global optimization problem, while Ref.2 is a MILP) gets values, on average, closed to those obtained by Ref.2 for the synthetic instances. However, for the case-study instances the situation is different and the average solution values and times move away from the optimal averages as the size of the instance increases. Note that for most of the synthetic instances, the optimal value is zero, i.e., the supply is perfectly balanced with the demand. Hence, neither shortages nor surplus exist due to the generation mechanism of these instances.

Regarding the heuristic algorithms, it can been observed from table 4 that EPIA and HYBA report values, on average very close, to the optimal ones for all the sizes of the synthetic instances. However, for the case-study instances, HYBA reports the best results among all the three heuristic algorithms (see table 3). Moreover, the performance of EPIA and FGPA significantly depends on the size of the instance. In terms of the average required time, FGPA requires less time for the case-study instances. This finding may be derived from the fact that the algorithm explores points not necessarily in the inducible region, which may help to approach the optimal solution in a faster manner. On the contrary, HYBA is the algorithm that requires the more time, but this may be obvious since it performs all the steps of EPIA and at each iteration it may solve upon four extra mathematical programs. Nevertheless, the registered times are acceptable for a problem of this nature. 
To compare the efficiency and the quality of the solutions obtained by the heuristic algorithms, the optimality gap is computed using the values obtained by Ref.2. Given that there are many optimal values equal to zero, the optimality gap (GAP) measures the relative deviation from the optimal value, and it is computed by the following formula:

$$
\mathrm{GAP}=\left|\frac{(\text { Optimal value })-(\text { Obtained value })}{(\text { Obtained value })}\right| \times 100 \%
$$

Also, an analogous formula is used to compute the relative savings in time $(\% t)$. That is, the reduction in computational time when solving the problem using the heuristics rather than Ref.2. A negative value indicates that the heuristic consumes more time than the exact reformulation. The formula used is presented next:

$$
\% \mathrm{t}=\frac{(\text { Required time by Ref. } 2)-(\text { Required time by a heuristic })}{(\text { Required time by a heuristic })} \times 100 \%
$$

The GAP and \%t average values obtained for both type of instances are shown in tables 5 and 6 , respectively.

Table 5: Evaluating the effectiveness of the heuristic algorithms in the case-study instances

\begin{tabular}{lrrrrrr}
\hline \multirow{2}{*}{ Size } & \multicolumn{2}{c}{ EPIA } & \multicolumn{2}{c}{ FGPA } & \multicolumn{2}{c}{ HYBA } \\
& GAP & $\% t$ & GAP & $\% t$ & GAP & $\% t$ \\
\hline $10 \times 10$ & $42.27 \%$ & $12 \%$ & $29.60 \%$ & $15 \%$ & $7.68 \%$ & $-48 \%$ \\
$25 \times 25$ & $40.15 \%$ & $746 \%$ & $11.70 \%$ & $534 \%$ & $1.67 \%$ & $190 \%$ \\
$25 \times 75$ & $53.78 \%$ & $1156 \%$ & $19.67 \%$ & $1101 \%$ & $7.04 \%$ & $445 \%$ \\
$50 \times 100$ & $54.29 \%$ & $3418 \%$ & $10.72 \%$ & $5418 \%$ & $6.02 \%$ & $1176 \%$ \\
$75 \times 125$ & $33.20 \%$ & $5269 \%$ & $12.26 \%$ & $10696 \%$ & $5.64 \%$ & $1499 \%$ \\
$150 \times 200$ & $19.29 \%$ & $9210 \%$ & $17.20 \%$ & $30206 \%$ & $4.92 \%$ & $3324 \%$ \\
\hline
\end{tabular}

Table 6: Evaluating the effectiveness of the heuristic algorithms in the synthetic instances

\begin{tabular}{lrrrrrr}
\hline \multicolumn{1}{c}{ Size } & \multicolumn{2}{c}{ EPIA } & \multicolumn{2}{c}{ FGPA } & \multicolumn{2}{c}{ HYBA } \\
& GAP & $\% t$ & GAP & $\% t$ & GAP & $\% t$ \\
\hline $10 \times 10$ & $6.67 \%$ & $79 \%$ & $10.00 \%$ & $42 \%$ & $0 \%$ & $69 \%$ \\
$25 \times 25$ & $0 \%$ & $1332 \%$ & $46.67 \%$ & $1038 \%$ & $0 \%$ & $1283 \%$ \\
$25 \times 75$ & $0 \%$ & $2189 \%$ & $50.00 \%$ & $1895 \%$ & $0 \%$ & $2192 \%$ \\
$50 \times 100$ & $0 \%$ & $13714 \%$ & $90.00 \%$ & $12176 \%$ & $0 \%$ & $14466 \%$ \\
$75 \times 125$ & $0 \%$ & $16773 \%$ & $90.00 \%$ & $14272 \%$ & $0 \%$ & $18485 \%$ \\
$150 \times 200$ & $0 \%$ & $11561 \%$ & $100.00 \%$ & $11377 \%$ & $0 \%$ & $12781 \%$ \\
\hline
\end{tabular}


HYBA is the algorithm that shows the best quality in the feasible solutions obtained. For the case study instances, the average optimality gap was lower than $8 \%$ for all the sizes (see table 5); while for the synthetic instances, the average optimality gap was zero (see table 6). FGPA presents a very good average gap in comparison with EPIA in the case-study instances, but this behavior is opposite in the synthetic instances. Concerning the savings in the required time, the three heuristic algorithms showed significant savings, which is improved as the size of the instance increases. FGPA is the one that evidence more savings for the case-study instances. It is convenient to mention that for the synthetic instances of size $10 \times 10$, HYBA showed a negative saving, this implies that Ref.2 was faster to solve these instances. But, this is expected since HYBA solves at least one linear model and one MILP model, and Ref.2 only solves one MILP model. In spite of that, the advantage of HYBA over the reformulation is evident for medium and large-size instances.

To support the latter findings, the number of optimal solutions obtained by each heuristic algorithm are displayed in table 7 . It can be seen from that table that HYBA is the algorithm that was able to obtain more optimal solutions. Also, it an be observed that FGPA obtained more optimal solutions for the case-study instances than EPIA, but for the synthetic instances the behavior was the opposite. These results confirm the suitability for combining both heuristic algorithms to create HYBA.

Table 7: Number of optimal solutions obtained for each heuristic algorithm

\begin{tabular}{llrrr}
\hline Instance & Size & EPIA & FGPA & HYBA \\
\hline \multirow{4}{*}{ Case-study } & $10 \times 10$ & 7 & 11 & 20 \\
& $25 \times 25$ & 0 & 12 & 19 \\
& $50 \times 75$ & 5 & 13 & 19 \\
& $75 \times 125$ & 1 & 13 & 15 \\
& $150 \times 200$ & 0 & 4 & 9 \\
& $10 \times 10$ & 28 & 27 & 1 \\
Synthetic & $25 \times 25$ & 30 & 16 & 30 \\
& $50 \times 100$ & 30 & 15 & 30 \\
& $75 \times 125$ & 30 & 3 & 30 \\
& $150 \times 200$ & 30 & 0 & 30 \\
\hline
\end{tabular}

\subsection{Using heuristic solutions to obtain the optimal}

The good performance of the heuristic algorithms lead us to analyze the idea of use the near-optimal obtained solutions as an input to Ref.2 seeking to enhance their process. By doing this, we expect to significantly reduce the computational time required to optimally solve an instance. The results obtained from this experimentation are shown in table 8 . The columns with label "Ref.2", "HYBA", and "Ref.2 w/initial sol." display the average 
required time for each solution scheme. The final column "Total" sums the time required for the hybrid algorithm and Ref.2 using an initial good solution. As it was expected, the advantages of using this scheme are more notorious for medium and large-size instances. The best example of this finding is given by the instances of size $150 \times 200$, in which the time was reduced from 2754.68 s to 586.53 s, and from 193.48 s to only 7.28 s, for the case-study and synthetic instances, respectively.

Table 8: Average required time when Ref.2 uses an initial heuristic solution

\begin{tabular}{clrrrr}
\hline Instance & \multicolumn{1}{c}{ Size } & \multicolumn{1}{c}{ Ref.2 } & HYBA & Ref.2 w/initial sol. & Total \\
\hline & $10 \times 10$ & 0.18 & 0.33 & 0.07 & 0.40 \\
& $25 \times 25$ & 1.86 & 0.82 & 0.39 & 1.21 \\
Case study & $25 \times 75$ & 4.36 & 1.54 & 0.80 & 2.34 \\
& $50 \times 100$ & 53.15 & 6.07 & 4.24 & 10.31 \\
& $75 \times 125$ & 268.89 & 20.24 & 19.73 & 39.97 \\
& $150 \times 200$ & 2754.68 & 196.38 & 390.15 & 586.53 \\
& $10 \times 10$ & 0.14 & 0.09 & 0.04 & 0.13 \\
& $25 \times 25$ & 1.43 & 0.12 & 0.10 & 0.22 \\
Random & $25 \times 75$ & 3.65 & 0.19 & 0.49 & 0.68 \\
& $50 \times 100$ & 35.52 & 0.33 & 1.15 & 1.48 \\
& $75 \times 125$ & 90.48 & 0.46 & 2.22 & 2.68 \\
& $150 \times 200$ & 193.48 & 1.95 & 7.28 & 9.23 \\
\hline
\end{tabular}

\section{Conclusions and research directions}

This paper studies a market regulation situation, in which the government controls the distribution of raw material and competes against private firms in the production of final commodities. In this market, the government has a privileged position since it determines its production and the amount of raw material offered to private firms and its goal is to balance the market. Although this type of market situation is common in some economies, to the best of our knowledge and after an intensive literature revision, we did not find a similar problem in the literature. The situation that motivated our paper comes from the actual situation in Mexican petrochemical industry with PEMEX from 1958 till 2014. However, this case is not exclusive from the petrochemical industry. Also in the agricultural industry, the Mexican government monopolized the production, imports, and distribution of fertilizers from 1970 to 1986 through the state-owned company named FERTIMEX [28]. The government also regulated the water supply for agricultural consumption by fixing prices, defining production goals, and restricting the land transactions [29]. In addition, this type of economic regulation has been applied in other countries. For example, in [3], it is stated that there is a tendency to use government instruments in developing countries, mainly in raw material industries. An indicator of the latter can be seen in the large amount of oil expropriations in the 70's (see [4], [5], [6]). Also, nationalizations occurred in Bolivia, Ecuador, Venezuela, and Russia in 2006 (see [6]). 
Motivated by the lack of models to analyze these type of markets, this paper develops a bilevel programming approach that fits naturally to the problem. To solve the bilevel problem herein proposed, two single-level reformulations are presented. The first reformulation uses the lower level necessary and sufficient optimality conditions to ensure the global optimum of the bilevel problem. As a result of this reformulation, a continuous non-linear problem is obtained. The second reformulation substitutes the above-mentioned conditions by the complementarity slackness conditions generating a MILP. These reformulations are able to solve optimally limited size instances. Therefore, three heuristic algorithms are proposed to find good quality feasible solutions in a reasonable computational time. The first two algorithms (EPIA and FGPA) are tailored for this problem, and exploits the fact that the polyhedron associated to the dual problem of the lower level remains the same for any leader's decision. The third algorithm (HYBA) is a combination of the previous ones.

Extensive computational experimentation is performed to test the exact and heuristic approaches proposed in this paper. Two sets of instances are used: case-study instances, that arise from the Mexican petrochemical industry, and synthetic instances adapted from the previous ones. As a result from the computational experimentation, it can be stated that the reformulation that applies the complementarity slackness constraints is more efficient for the tested instances. On the other hand, the performance of EPIA and FGPA strongly depends on the characteristics of the instance. In addition, the hybridization of both heuristic algorithms (HYBA) yields to the best results in terms of effectiveness and solutions quality. Moreover, HYBA found the optimal solution for all the synthetic instances, and obtained optimality gaps less than $8 \%$ in the case-study ones. It is worth to mention that HYBA found the optimal value in at least $50 \%$ for the first four types of instance sizes.

Finally, it can be concluded from our computational experiment, that the most efficient manner to find optimal solutions for the bilevel problem is by obtaining a good quality feasible solution through HYBA and then using it as an initial solution to solve Ref.2. By doing this, a significant computational time reduction is achieved.

Note that this bilevel problem may have multiple optimal solutions, each of them offers different alternatives to the upper level decision-maker (government) for producing and offering raw material to the lower level decision-makers (private firms). Therefore, this characteristic is economically worthy to be explored. Another possible research direction is to neglect, in the model, the assumption there exists an organism that manages the cooperation among private firms. Hence, a new element will emerge in the model since competition among private firms will also exist. As a result of this, a bilevel problem with one leader and multiple followers will appear. Then, in a natural way, a Stackelberg game in which the follower's problem consists of a generalized Nash game fits to analyze this situation. 


\section{Acknowledgements}

José-Fernando Camacho-Vallejo has been partially supported by the program of professional development of professors with the grant PRODEP/511-6/17/7425 for research stays during his sabbatical year. Justo Puerto has been partially supported by Spanish/FEDER grants number MTM2016-74983-C02-01 (MINECO). Martine Labbé has been partially supported by the Fonds de la Recherche Scientique - FNRS under Grant(s) no PDR T0098.18.

\section{References}

[1] E. G. Wiens, Government firm regulation of a vertically integrated industry, Carleton Economic Paper (1978) 78-109.

[2] J. E. Stiglitz, Regulation and failure, in: D. Moss, J. Cisternino (Eds.), New Perspectives on Regulation, Tobin Project, 2009.

[3] R. Vernon, Uncertainty in the resource industries: the special role of state-owned enterprises, in: D. W. Pearce, H. Siebert (Eds.), Risk and the Political Economy of Resource Development, Springer, 1984, pp. 207-223.

[4] S. J. Kobrin, The nationalisation of oil production, 1918-80, in: D. W. Pearce, H. Siebert (Eds.), Risk and the Political Economy of Resource Development, Springer, 1984, pp. 137-164.

[5] M. S. Minor, The demise of expropriation as an instrument of ldc policy 1980-1992, Journal of International Business Studies 25 (1) (1994) 177-188.

[6] S. Guriev, A. Kolotilin, K. Sonin, Determinants of nationalization in the oil sector: A theory and evidence from panel data, The Journal of Law, Economics, and Organization 27 (2) (2011) 301-323.

[7] W. C. Merrill, N. Schneider, Government firms in oligopoly industries: A short-run analysis, The Quarterly Journal of Economics 80 (3) (1966) 400-412.

[8] B. Blackwell, The state and the macro-industrial economy: Lessons from the aerospace sector, Omega 14 (6) (1986) 421-442.

[9] R. G. Harris, E. G. Wiens, Government enterprise: An instrument for the internal regulation of industry, The Canadian Journal of Economics 13 (1) (1980) 125-132.

[10] M. R. Sertel, Regulation by participation, Journal of Economics 48 (2) (1988) 111-134.

[11] H. Cremer, M. Marchand, J.-F. Thisse, The public firm as an instrument for regulating an oligopolistic market, Oxford Economic Papers 41 (2) (1989) 283-301.

[12] G. De Fraja, F. Delbono, Game theoretic models of mixed oligopoly, Journal of Economic Surveys 4 (1) (1990) $1-17$.

[13] G. De Fraja, F. Delbono, Mixed oligopoly: An overview, Tech. rep., Quaderni-Working Paper DSE (1988).

[14] L. Nett, Mixed oligopoly with homogeneous goods, Annals of Public and Cooperative Economics 64 (3) (1993) 367-393.

[15] E. Z. Demirci, N. K. Erkip, Designing an intervention strategy for public-interest goods: The california electric vehicle market case, Omega 69 (2017) 53-69.

[16] G. Whittaker, R. Färe, S. Grosskopf, B. Barnhart, M. Bostian, G. Mueller-Warrant, S. Griffith, Spatial targeting of agri-environmental policy using bilevel evolutionary optimization, Omega 66 (2017) 15-27.

[17] S. Dempe, Bilevel programming: A survey, Dekan der Fak. für Mathematik und Informatik, 2003.

[18] J. F. Bard, Practical Bilevel Optimization, Nonconvex Optimization and Its Applications, Springer US, 1998.

[19] Reglamento de la ley reglamentaria del artículo 27 constitucional en el ramo del petróleo, en materia de petroquímica, Diario Oficial, méxico, 9 de febrero de 1971.

[20] Ley reglamentaria del artículo 27 constitucional en el ramo del petróleo, Diario Oficial, méxico, 29 de noviembre de 1958. 
[21] Acuerdo que dispone que corresponde a petróleos mexicanos u organizaciones o empresas subsidiarias o asociadas a la misma institución, la elaboración de diversos productos de petroquímica básica, Diario Oficial, méxico, 9 de abril de 1960.

[22] Ley orgánica de petróleos mexicanos y organismos subsidiarios, Diario Oficial de la Federación, méxico, 16 de julio de 1992.

[23] Reglamento de la ley reglamentaria del artículo 27 constitucional en el ramo del petróleo, Diario Oficial, méxico, 25 de agosto de 1959.

[24] Resolución que clasifica los productos petroquímicos que se indican, dentro de la petroquímica básica o secundaria, Diario Oficial de la Federación, méxico, 13 de octubre de 1986.

[25] Resolución que clasifica los productos petroquímicos que se indican, dentro de la petroquímica básica o secundaria, Diario Oficial de la Federación, méxico, 15 de agosto de 1989.

[26] Resolución que clasifica los productos petroquímicos que se indican, dentro de la petroquímica básica o secundaria, Diario Oficial de la Federación, méxico, 17 de agosto de 1992.

[27] Decreto por el que se reforma la ley reglamentaria del artículo 27 constitucional en el ramo del petróleo, Diario Oficial de la Federación, méxico, 13 de noviembre de 1996.

[28] A. De Janvry, I. F. for Agricultural Development, I.-A. I. for Cooperation on Agriculture, Reformas del sector agrícola y el campesinado en México, Estrategias para mitigar la pobreza rural en América Latina y El Caribe, Fondo Internacional de Desarrollo Agrícola, 1995.

[29] S. Johnson, La transferencia del manejo de la irrigación en México: una estrategia para lograr la sostenibilidad de los distritos de riego., IWMI Research Report, Instituto Internacional del Manejo de la Irrigación, 1997. 